\title{
A Neural System for Faithful Color Reproduction in Industrial Printing Processes
}

\author{
Beatrice Lazzerini, and Francesco Pistolesi \\ Department of Information Engineering, University of Pisa \\ Address: Largo L. Lazzarino, 1 - 56122 Pisa, ITALY \\ Email: b.lazzerini@iet.unipi.it, f.pistolesi@iet.unipi.it
}

\begin{abstract}
Printing is a widespread industrial process. Manufacturers of colored products are expected to maintain high levels of color quality to perfectly satisfy the customers' requirements. The rendering of colors is visually checked by experienced workers, who may though show different color sensitiveness, depending, e.g., on perceptual, cognitive and cultural aspects. This often results in products that fail to faithfully reproduce what the customer asked for, with negative consequences for companies, as well as huge financial losses. This paper describes a neural network-based system to objectively check how faithfully colors are reproduced by an industrial printing process. The system considers a master color, then compares it to a copy, and returns an objective degree of color fidelity of the copy to the master. The neural system was trained and tested in a real-world case study by using a huge quantity of color pairs taken from the $\mathbf{L}^{*} \mathbf{a}^{*} \mathbf{b}^{*}$ color space. Highly accurate results were achieved. The strengths of the system are that it can measure the difference of colors in a way that is incredibly close to that perceived by the human eye, and the fact that it can do that canceling the color distortion phenomena that may occur in the human vision.
\end{abstract}

\section{INTRODUCTION}

Printing is pervasive nowadays. Just think about the huge amount of customized boxes and wrappings that come to our homes every day. There are many industries where printing processes are involved, such as packaging, commercial and decorative printing. The global printing industry gets stunning revenues and is forecast to reach $\$ 980$ billion by 2018 [1].

Digital printing processes are incredibly sophisticated today, and require frequent calibrations to control the color fidelity. This happens because these processes are subject to various phenomena that influence the rendering of color. If a production batch-for example thousands of food packages-is not chromatically homogeneous, or colors are different from how the customer perceived those colors when signing the supply contract, negative consequences may occur for companies, such as huge financial losses. Since the customer requirements are often not easy to satisfy, managing the color fidelity is a key problem, especially because customers rarely purchase defective products. The only way to convince them to do that is to discount the price. But unforeseen discounts may lead to a loss for the company.

The color rendering and homogeneity of a printing process are today machine-assisted, but are still mainly based on visual

This work was co-funded by the Tuscany Region and Macs Tech S.r.1., under the POR FSE 2014-2020 programme. inspections that are performed by experienced employees who are responsible for the color accuracy. Unfortunately, these workers may have different color sensitiveness, depending on perceptual, cognitive and cultural aspects. Typical aspects include mood, age, focus ability, color matching, lightness, and the fact that warm or cool colors are being observed [2]. Therefore, visual checks may result in products that fail to accurately reproduce the colors the customer asked for, or even worse, the printed colors may be different from an item to another, within the same batch.

Over the last decades, the $\mathrm{CIE}^{1}$ has proposed the $\mathrm{L}^{*} \mathrm{a}^{*} \mathrm{~b}^{*}$ color space and different formulas to measure the difference between two colors [3], [4]. The most used is the L*a*b* distance or $\Delta E$, which was introduced in 1976 and is still widely used in industry and research. This formula has some shortcomings that make it unable to exactly map the difference that the human eye perceives when comparing two colors.

That is why the CIE has introduced more complicated formulas over the years, in particular the $\Delta E_{94}$ and $\Delta E_{00}$, released in 1994 and 2000, respectively. These measures introduce several parameters and weighting functions, but lack of complete visual uniformity [5].

Many advanced techniques have recently been proposed to address the problem of guaranteeing that a color is reproduced as accurately as possible. For instance, there are recent studies on color calibration [6], and on the appearance of colors on electronic devices when used under high ambient lighting conditions [7]. Other approaches investigate the effect of the paper properties on the color reproduction in digital printing [8], or propose visual brightness functions to enhance the contrast and saturation of rendered images [9]. From the industry perspective, there are several applications to control and guarantee that colors are reproduced accurately. Some of the more recent include the spectrophotometric analysis of the color of ceramic restorations in dentistry [10], and pattern matching methods for the quality control in automotive metallic coatings [11].

This paper focuses on the printing industry, and proposes a neural system to measure the difference between two printed colors as closely as possible to how this difference is perceived

\footnotetext{
${ }^{1}$ The Commission Internationale de l'Eclairage (CIE) is the international authority of light, illumination, color, and color spaces.
} 
by the human eye. The system is made up of two multi-layer perceptron neural networks (comparators) that take the $\mathrm{L}^{*} \mathrm{a} * \mathrm{~b}^{*}$ coordinates of two colors and return their color difference. The difference is measured objectively, thus canceling the effects of those phenomena that alter the perception of colors, which are typical of the human vision, like, e.g., the ones that modify the perceived difference of colors as a consequence of the observer's mood and level of fatigue.

The paper is organized as follows. Section II contains a background on color and color perception. Section III describes the proposed way to fix the deficiencies of $\Delta E$. Section IV presents the neural comparators and the developed system. Section V draws the conclusions.

\section{BACKGROUND}

Color is a physiological sensation that our brain uses to recognize the objects around us. The color sensation basically occurs according to the following four steps:

1) a light source emits a light;

2) an object reflects this light by modifying it according to its surface characteristics;

3) the human eye detects this reflected light and turns it into a stimulus;

4) the brain processes this stimulus and generates the chromatic sensation.

\section{A. What is light?}

Light is an electromagnetic radiation. The portion that the human eye can sense (commonly known as visible light) is the radiation with wavelengths ranging from $\sim 380 \mathrm{~nm}$ (violet) to $\sim 800 \mathrm{~nm}$ (red).

A light source is something that emits light, such as the neon lamps in office buildings. A light source emits different amount of energy (i.e. power) at each wavelength $\lambda$ of the visible color spectrum, thus forming its spectral power distribution, denoted with SPD or $S(\lambda)$.

\section{B. Standard illuminants}

The CIE has standardized the different light sources by the amount of energy emitted at each wavelength (i.e. the SPD). Unlike a light source, an illuminant is not a physical object, but rather a representation of the SPD curve of a light, e.g. by means of a graph (examples are in Fig. 1). SPDs are generally represented as relative SPDs, normalizing in $[0,100]$ the amount of energy emitted.
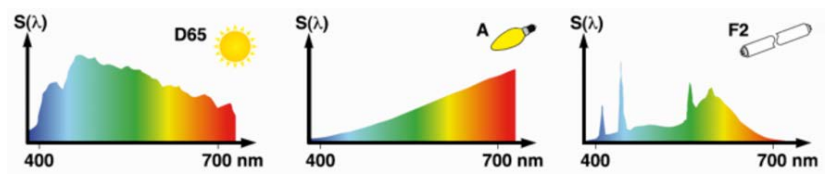

Fig. 1. SPDs of three standard illuminants: daylight (D65), incandescent bulb (A), cool white fluorescent lamp (F2).

\section{Object}

When a light strikes the surface of the objects, they absorb and reflect specific amounts of energy at each wavelength. In the case of a red object, for instance, the red part of the visible spectrum is reflected almost completely, whereas the energy at the other wavelengths is mainly absorbed.

The percentage of incident electromagnetic power that is reflected at each wavelength is called reflectance. The plot of the reflectance of an object as a function of the wavelength is called spectral reflectance curve (see Fig. 2).

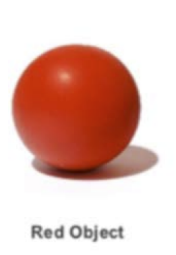

Fig. 2. Spectral reflectance curve of a red ball.

\section{Observer}

When illuminating an object with a light source, the reflected light coming from the object reaches an observer (e.g. the human eye). This reflected light has an SPD, which is referred to as reflected SPD or rSPD.

The rSPD is obtained by multiplying the energy that comes from the light source by the reflectance of the object for each $\lambda \in[380 \mathrm{~nm}, 800 \mathrm{~nm}]$, as shown in Fig. 3 .

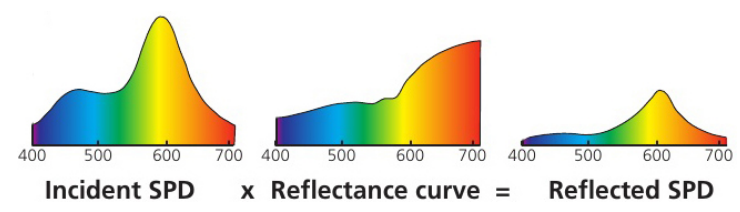

Fig. 3. Reflected SPD (rSPD) obtained multiplying the relative SPD of the light source (incident SPD) by the reflectance of the object.

\section{E. Color perception}

Given the SPD of a light that reaches the retina, three types of cones jointly generate a 3-tuple $(\rho, \gamma, \beta)$ called the tristimulus response. Let $S(\lambda)$ be the SPD, where $\lambda$ is the wavelength, and let $\bar{\rho}(\lambda), \bar{\gamma}(\lambda)$ and $\bar{\beta}(\lambda)$ be the sensitivity functions of the three types of cones. The response can then be computed by integrating the products of wavelength intensity and cone sensitivity over the visible spectrum, i.e.,

$$
\begin{gathered}
(\rho, \gamma, \beta)= \\
\left(\int_{\lambda_{l}}^{\lambda_{u}} S(\lambda) \bar{\rho}(\lambda) d \lambda, \int_{\lambda_{l}}^{\lambda_{u}} S(\lambda) \bar{\gamma}(\lambda) d \lambda, \int_{\lambda_{l}}^{\lambda_{u}} S(\lambda) \bar{\beta}(\lambda) d \lambda\right)
\end{gathered}
$$

where $\lambda_{l}=380 \mathrm{~nm}$ and $\lambda_{u}=800 \mathrm{~nm}$. Our color perception is determined by this 3 -tuple response. Functions $\bar{\rho}(\lambda), \bar{\gamma}(\lambda)$ 
and $\bar{\beta}(\lambda)$ are called color matching functions and have been determined through experiments.

Given three color matching functions, any visible color is represented using a 3-tuple.

\section{F. CIE RGB color space}

RGB is a convenient color model to represent colors in computer graphics, as the human visual system works like an RGB color space, though not quite identical. The $(R, G, B)$ 3 -tuple that describes a color is

$$
\begin{aligned}
& (R, G, B)= \\
& \left(\int_{\lambda_{l}}^{\lambda_{u}} S(\lambda) \bar{r}(\lambda) d \lambda, \int_{\lambda_{l}}^{\lambda_{u}} S(\lambda) \bar{g}(\lambda) d \lambda, \int_{\lambda_{l}}^{\lambda_{u}} S(\lambda) \bar{b}(\lambda) d \lambda\right)
\end{aligned}
$$

where $\bar{r}(\lambda), \bar{g}(\lambda)$ and $\bar{b}(\lambda)$ are the (experimentally obtainable) color matching functions shown in Fig. 4. The $(R, G, B) 3$ tuple uniquely determines a visible color, given a light source.

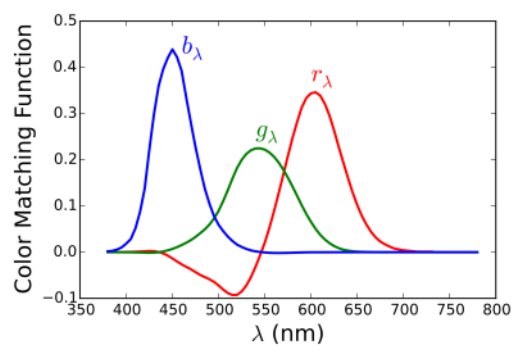

Fig. 4. Intensity curves of source lights $\mathrm{R}, \mathrm{G}$ and $\mathrm{B}$. When used as color matching functions, we get the CIE RGB color space.

\section{G. CIE $L^{*} a * b *$ color space}

CIE $\mathrm{L} * \mathrm{a} * \mathrm{~b} *$ is a color space that describes all perceivable colors in three dimensions: $\mathrm{L}^{*}$ for lightness; $\mathrm{a}^{*}$ and $\mathrm{b}^{*}$ for the color opponents green-red and blue-yellow, respectively. The CIE L*a*b* system thus describes and orders colors on the basis of the color opponent theory, which says that colors cannot be perceived as both red and green at the same time, or yellow and blue at the same time. Colors can instead be perceived as one of the following combinations: red and yellow; red and blue; green and yellow; green and blue.

The CIE L*a*b* color space was introduced as a perceptually uniform color space, where the difference between two colors at a given distance from each other is perceived the same way, no matter the region they come from. The CIE L*a*b* color space was purposely designed to approximate the human vision as close as possible.

Given a color, the $\mathrm{L}^{*}, \mathrm{a}^{*}$ and $\mathrm{b}^{*}$ coordinates represent the color lightness, the position between red/magenta and green, and the position between yellow and blue, respectively. In particular, $\mathrm{L}^{*}=0$ is the darkest black and $\mathrm{L}^{*}=100$ indicates diffuse white; negative values of $\mathrm{a}^{*}$ indicate green, whereas positive values indicate magenta; negative values of $b^{*}$ indicate blue and positive values indicate yellow.

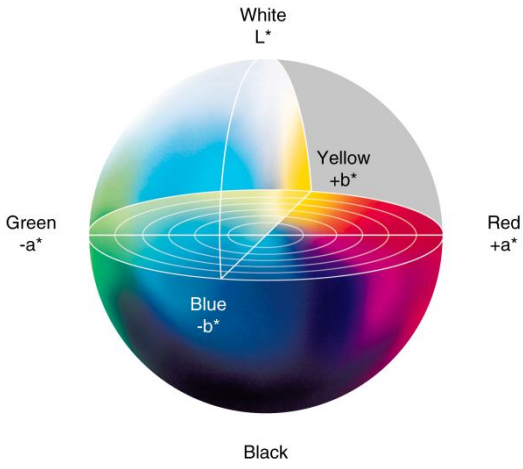

Fig. 5. The CIE L*a*b* color space.

\section{H. Measuring the difference of colors}

As the CIE L*a*b* is a perceptually uniform color space, the difference between two colors can be measured by using the Euclidean distance. This way of measuring the difference of colors was introduced by the CIE in 1976 and is called $\Delta E$ (or L*a*b* distance). Formally, if $\left(L_{1}^{*}, a_{1}^{*}, b_{1}^{*}\right)$ and $\left(L_{2}^{*}, a_{2}^{*}, b_{2}^{*}\right)$ are two colors in the CIE L*a*b* color space, their difference is:

$$
\Delta E=\sqrt{\left(L_{2}^{*}-L_{1}^{*}\right)^{2}+\left(a_{2}^{*}-a_{1}^{*}\right)^{2}+\left(b_{2}^{*}-b_{1}^{*}\right)^{2}} .
$$

This measure has some drawbacks. For example, when visually comparing a pair of colors that come from the dark region of the $\mathrm{L}^{*} \mathrm{a} * \mathrm{~b} *$ space-look at the bottom area of the sphere in Fig. 5,- - they are perceived as the same color even when their $\Delta E$ is very high. In other regions, this value of $\Delta E$ characterizes two colors that are completely different. There are other zones of the $L^{*} a^{*} b^{*}$ space where the same happens, for example the blue-violet region.

Over the last decades, the CIE has proposed several refinements of $\Delta E$. Even though these more recent versions better map the human vision, they are particularly complicated and retain some drawbacks. This work thus tries to start from Eq. (3) and aims to improve its accuracy, thereby fixing the drawbacks.

\section{IMPROVING $\Delta E$}

\section{A. Overview and motivation}

Equation (3) calculates the difference between two colors that is perceived by the human eye, and is thought to return values that are lower than 2 when two colors are hard to distinguish by the human eye. The higher the value of $\Delta E$, the more different two colors should be perceived.

Unfortunately, this does not hold anywhere one picks two colors in the $\mathrm{L}^{*} \mathrm{a}^{*} \mathrm{~b} *$ space. There are in fact some regions where the difference is less perceptible to the human eye. Imagine the $\mathrm{L}^{*} \mathrm{a} * \mathrm{~b} *$ sphere as a series of overlapped circles, as Fig. 6 shows. Looking at the figure, it is easy to understand the problem. When varying the value of $L^{*}$ (i.e., the brightness), the difference perceived comparing two given colors picked from the same region of each of the three slices changes. For example, when $L=0$ (the dark colors in the slice at the 


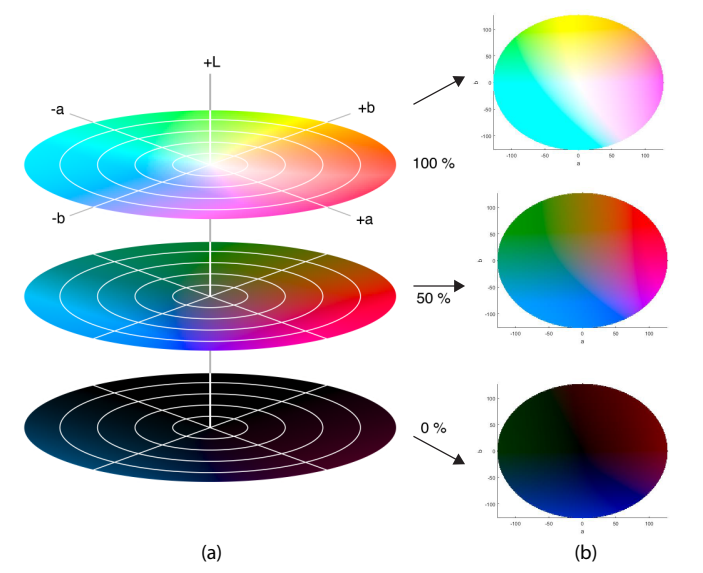

Fig. 6. Front view (a) and top view (b) of different horizontal slices of the $\mathrm{L}^{*} \mathrm{a} * \mathrm{~b}^{*}$ space.

bottom on the right-hand side of Fig. 6), it is easy to see that the difference is less perceptible, so here two colors with $\Delta E>2$ are the same to the human eye.

Another problem of the $\mathrm{L}^{*} \mathrm{a}^{*} \mathrm{~b}^{*}$ distance is that the same drawback described before (i.e., high value of $\Delta E$ for fairly undistinguishable colors) occurs in other regions, and is not completely due to the level of brightness of the two colors compared. Such regions are, for example, the gray region and the region that contains the colors that range from blue to violet. This means that the whole $\mathrm{L}^{*} \mathrm{a} * \mathrm{~b}^{*}$ space must be sampled in order to investigate and solve these problems that involve the hue of colors. In order to measure the difference of colors in a way that is perceptually uniform in the whole $\mathrm{L}^{*} \mathrm{a}^{*} \mathrm{~b}^{*}$ space, the $\mathrm{L}^{*} \mathrm{a}^{*} \mathrm{~b}^{*}$ distance has thus to be modified.

The next sections describe the two modifications that were made to get a measure that is very close to how the human eye perceives the difference of colors.

\section{B. First step: fixing the drawbacks due to changing the brightness}

Consider two colors whose coordinates in the $\mathrm{L}^{*} \mathrm{a} * \mathrm{~b} *$ color space are $\left(L_{1}^{*}, a_{1}^{*}, b_{1}^{*}\right)$ and $\left(L_{2}^{*}, a_{2}^{*}, b_{2}^{*}\right)$, respectively. In order to fix the mismatch between the $L^{*} a^{*} b^{*}$ distance and the perceived difference between two colors when varying the brightness, a series of visual experiments was made. These experiments led to the definition of the piecewise regularization function shown in Fig. 7. The slope of each segment was determined as follows.

Ten partitions (precisely, thick horizontal slices) of the $\mathrm{L}^{*} \mathrm{a} * \mathrm{~b} *$ sphere were first considered, containing, respectively, the colors with brightness ranging from 0 to 10,11 to 20 and so on. A uniform set of color pairs with $\Delta E \in\{2,3,4\}$ were considered in the volume within each slice. Ten observers were then asked to sequentially look at all these color pairs, and classify each of them as containing equal, slightly different or different colors. The number of votes of each class was counted for each color pair with $\Delta E=2$. If the majority of the votes was equal the slope was set to zero. The slope was otherwise increased/decreased by $5 \%$ and the test was done again. The same procedure was repeated for color pairs with $\Delta E=3$ and $\Delta E=4$. In these cases, the highest number of votes to maintain the $\Delta E$ unaltered was expected in the slightly different and different classes, respectively.

The result of the experiments was that the $\mathrm{L}^{*} \mathrm{a} * \mathrm{~b} *$ distance satisfactorily matches the perceived difference of colors for values that range from $L^{*}=30$ to $L^{*}=80$. Elsewhere, the distance along the $L^{*}$ dimension does not map the perception of the human eye uniformly, and thus needs to be rescaled. In order to adjust the distance along the $\mathrm{L}^{*}$ dimension, the following function is proposed:

$$
\Delta L_{\text {mod }}^{*}=\int_{L_{1}^{*}}^{L_{2}^{*}} \psi\left(L^{*}\right) d L^{*}
$$

where $\psi\left(L^{*}\right):[0,100] \rightarrow[0,1]$ is the piecewise regularization function that was experimentally obtained as explained before. The function aims to make it true that values of $\mathrm{L}^{*} \mathrm{a}^{*} \mathrm{~b}^{*}$ distance lower than 2 actually correspond to pairs of colors that are indistinguishable to the human eye and vice versa, wherever one picks two colors with the same brightness in the $\mathrm{L}^{*} \mathrm{a}^{*} \mathrm{~b}^{*}$ space. Higher values of $\psi\left(L^{*}\right)$ represent a higher

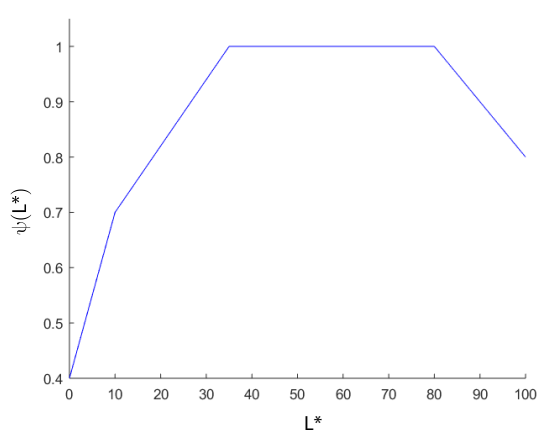

Fig. 7. Piecewise regularization function to fix the brightness nonuniformity of the $\mathrm{L}^{*} \mathrm{a}^{*} \mathrm{~b} *$ distance.

sensitivity - the difference of colors is easier to perceive, - on the other hand, lower values represent a lower sensitivity. The value obtained by Eq. (4) is then used as a substitute for term $\left(L_{2}^{*}-L_{1}^{*}\right)$ in Eq. (3). This leads to the definition of $\Delta E^{\text {mod }}$, whose expression is:

$$
\Delta E_{1,2}^{\text {mod }}=\sqrt{\int_{L_{1}^{*}}^{L_{2}^{*}} \psi\left(L^{*}\right) d L^{*}+\left(a_{2}^{*}-a_{1}^{*}\right)^{2}+\left(b_{2}^{*}-b_{1}^{*}\right)^{2}} .
$$

Note that when considering two colors $c_{1}$ and $c_{2}$ with $\psi\left(L_{1}^{*}\right)=$ $\psi\left(L_{2}^{*}\right)=1$, nothing changes with respect to the original $\mathrm{L}^{*} \mathrm{a} \mathrm{b}^{*}$ distance, as the difference calculated using Eq. (3) accurately maps the human perception of colors.

\section{Second step: refining $\Delta E^{\text {mod }}$ by including the human perception of hues}

In order to improve the accuracy of $\Delta E^{\text {mod }}$ when varying the hue, a perceptual information was incorporated into the formula, using a product. With the help of a developed tool equipped with an easy-to-use graphic interface, a similarity 
level in \{equal, almost equal, slightly different, different, definitely different $\}$ was assigned to each copy of a master. A number $k \in \mathbb{R}$ was then associated with each similarity level. This number is multiplied by $\Delta E^{\text {mod }}$ in order to get the similarity rate $\sigma$. Considered two colors whose coordinates in the $\mathrm{L}^{*} \mathrm{a}^{*} \mathrm{~b}^{*}$ color space are $\left(L_{1}^{*}, a_{1}^{*}, b_{1}^{*}\right)$ and $\left(L_{2}^{*}, a_{2}^{*}, b_{2}^{*}\right)$, respectively, the similarity rate is:

$$
\sigma_{1,2}=k \cdot \sqrt{\int_{L_{1}^{*}}^{L_{2}^{*}} \psi\left(L^{*}\right) d L^{*}+\left(a_{2}^{*}-a_{1}^{*}\right)^{2}+\left(b_{2}^{*}-b_{1}^{*}\right)^{2}} .
$$

The value of $k$ was chosen with the aim to increase/decrease the value of $\Delta E^{\text {mod }}$, thus refining what previously done with $\Delta E^{\text {mod }}$, thereby making $\sigma$ as close as possible to the way the human eye perceives the difference of colors. The values of $k$ that were experimentally found to refine $\Delta E^{\text {mod }}$ are:

- $k=0.5$ when master and copy are considered as equal;

- $k=0.8$ when the two colors are judged almost equal;

- $k=1$ when the two colors seem slightly different;

- $k=1.2$ when the two colors are judged different;

- $k=2$ when the colors are judged definitely different.

\section{THE NEURAL COMPARATOR}

\section{A. Overview}

This section describes a system made up of two multilayer perceptron (MLP) neural networks that were designed and trained to compare two colors $c_{1}$ and $c_{2}$, and return their $\Delta E_{1,2}^{\text {mod }}$ and $\sigma_{1,2}$, respectively.

The system was developed as a MATLAB tool with a user-friendly graphic interface. The tool was designed to generate a set of color pairs that are made up of a master and a copy, in order to simulate what actually happens when reproducing colors in industrial printing processes. The tool also allows to make comparisons between colors and quantify their difference. This incorporates the human perception of colors into the data.

This section also describes the details of the MLP neural networks, and the choices made to achieve the best results.

\section{B. Dataset}

The dataset is made up of 1232 reflectance spectra, each relative to a matt color patch (master). These colors, shown in Fig. 8, were chosen because they are colors that are actually used in a real-world industrial scenario.

Reflectance spectra are sampled with $1 \mathrm{~nm}$ step, from 380 $\mathrm{nm}$ to $800 \mathrm{~nm}$, thus collecting 421 samples per color patch. The dataset also contains the RGB and $\mathrm{L}^{*} \mathrm{a} * \mathrm{~b} *$ coordinates of each spectrum, calculated using the D65 illuminant (daylight).

The experiments described in this section were purposely thought and carried out to show how the proposed system actually works in a real-world case study. Even though the number of colors in the dataset may seem not so high to properly deal with the problem, these colors were in fact sufficient to demonstrate that the system works well and can really help reproduce colors faithfully in a real-world case

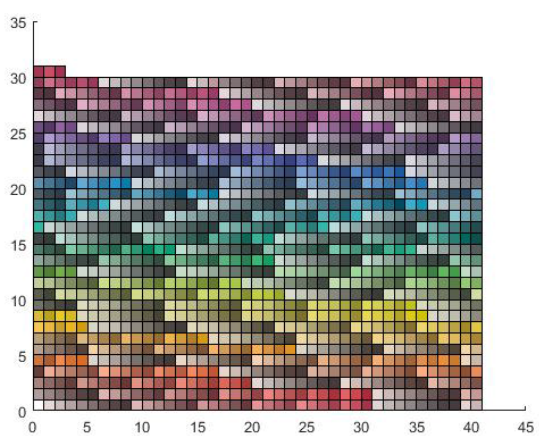

Fig. 8. Dataset of master color patches.

study related to a key industry, like packaging. Further works are in progress to investigate the use of the system with wider sets of colors and different material finishings.

\section{Generating reproductions of a color (copies)}

The developed system first asks how many masters to consider. Given a master $m$, the system can generate copies $c$ whose distance from the master (i.e., $\Delta E_{m, c}$ ) falls within $\left(\Delta E^{\min }, \Delta E^{\max }\right]$. When generating copies, the values of $\Delta E^{\min }$ and $\Delta E^{\max }$ are chosen according to one of the following criteria:

1) very close to the master, with $0.5<\Delta E_{m, c} \leq 2$, so copies that are almost equal to the master;

2) close to the master, with $2<\Delta E_{m, c} \leq 3$, these are copies that may be either equal or very similar to the master;

3) a little far from the master, with $3<\Delta E_{m, c} \leq 5$, these copies are very likely to be different from the master, but may be equal in specific cases;

4) far away from the master, with $5<\Delta E_{m, c} \leq 10$, i.e., copies that are very different from the master.

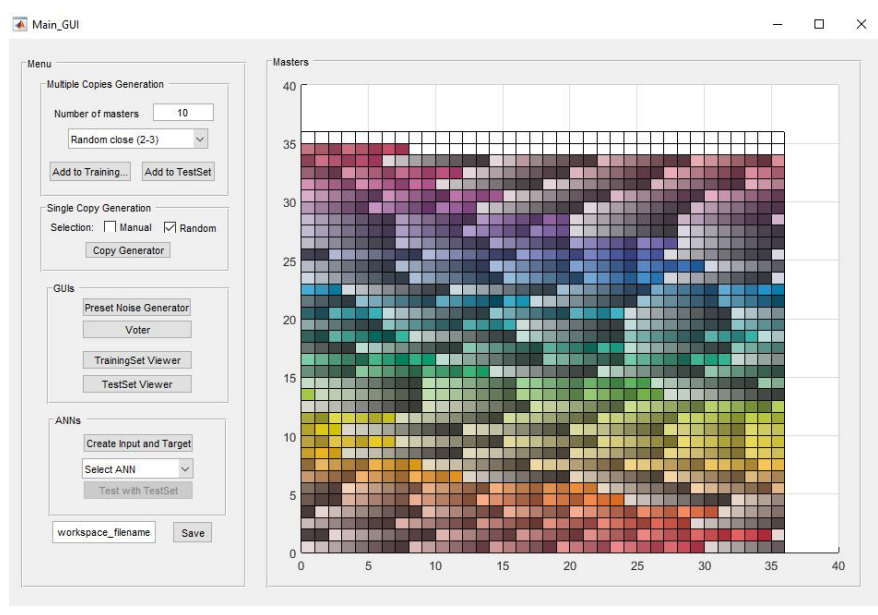

Fig. 9. The graphic interface to generate copies based on the $\mathrm{L}^{*} \mathrm{a} * \mathrm{~b} *$ distance.

Let $M$ be the chosen number of masters to put in set $\mathbb{M}$. If the user does not choose the whole dataset (i.e., $M<1232$ ), the system selects $M$ masters that are uniformly distributed in 


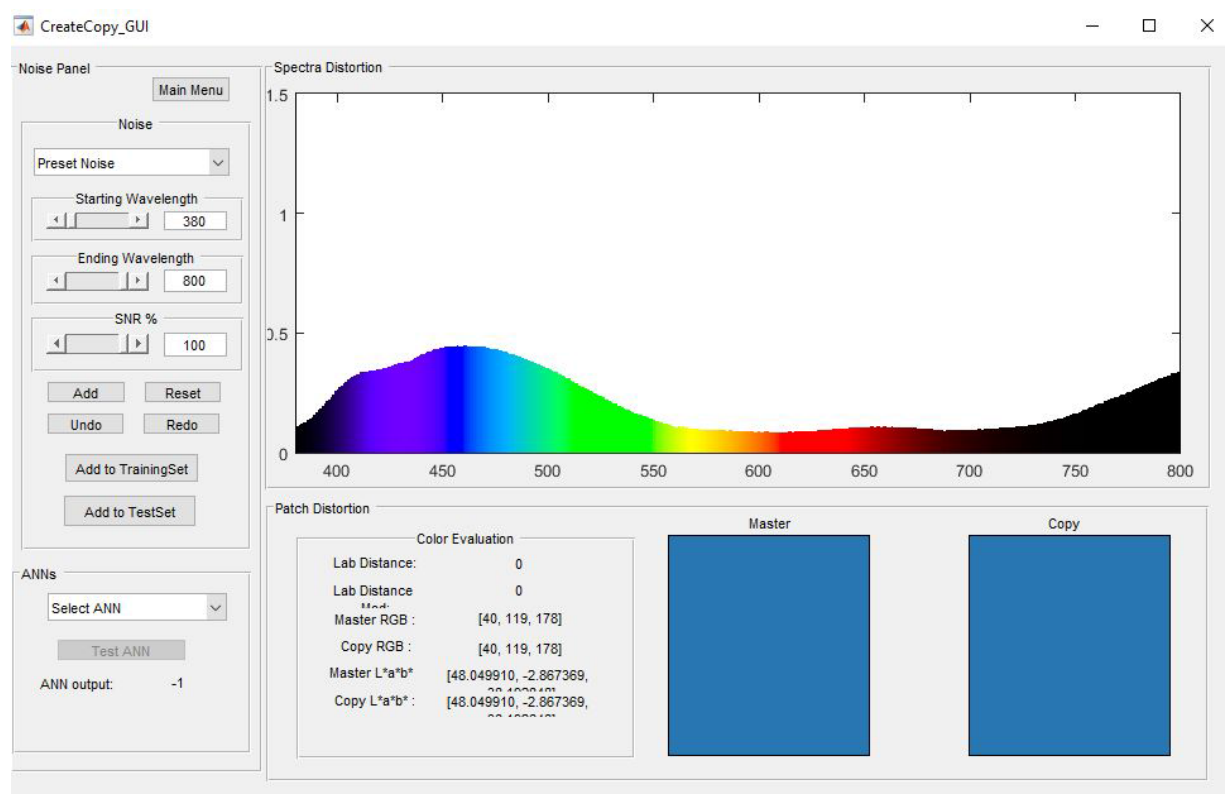

Fig. 10. The graphic interface to generate copies based on perturbing the spectrum with a Gaussian white noise.

the $\mathrm{L}^{*} \mathrm{a} * \mathrm{~b} *$ sphere, and these masters are then put into $\mathbb{M}$. For each master $m \in \mathbb{M}$, the system generates four copies $c_{1}^{m}, c_{2}^{m}$, $c_{3}^{m}$ and $c_{4}^{m}$. The distance of each copy from the master-and the other parameters - can be chosen using the corresponding drop-down menu on the left-hand side of Fig. 9.

From an operational point of view, given a master color $m \in$ $\mathbb{M}$, the system first generates a random number $u \sim \mathcal{U}(0,1)$, where $\mathcal{U}(0,1)$ denotes the uniform probability distribution in $[0,1]$. This number is then multiplied by the chosen $\Delta E^{\max }$. The system thus considers a spherical shell in the $L^{*} a^{*} b^{*}$ space, whose center is the master. This spherical shell is then divided into four quarters. The system then moves along the $\mathrm{a}^{*}$ and $\mathrm{b}^{*}$ dimensions, in order to obtain a copy in each quadrant of the spherical shell. We chose not to consider the $\mathrm{L}^{*}$ dimension at this stage because it is already included in the $\Delta E$, so the generated copies may have different $\mathrm{L}^{*}$ with respect to the master.

Copies of a given master can also be generated by perturbing its spectrum using a Gaussian white noise. This is helpful when desiring copies that are particularly close to the master, or when we aim to obtain copies that differ from the master in specific hues. This kind of copies can easily be generated by the developed tool by means of the appropriate interface that is shown in Fig. 10. As can be seen on the left-hand side of the figure, the user can easily select the region (i.e., the range of wavelengths) where the noise is added, and the signal noise ratio (SNR). The other areas on the right-hand side of the GUI show, at the top, the obtained spectrum, and at the bottom, from left to right, the color evaluation of master and copy (i.e., their $\mathrm{L}^{*} \mathrm{a} * \mathrm{~b}^{*}$ distance $\Delta E, \mathrm{~L}^{*} \mathrm{a} * \mathrm{~b} *$ distance modified $\Delta E^{\text {mod }}$, RGB and $\mathrm{L}^{*} \mathrm{a} * \mathrm{~b} *$ coordinates) and finally the master and copy patches.

\section{First comparator: reproducing $\Delta E^{\bmod }$}

The first neural comparator takes the $\mathrm{L}^{*} \mathrm{a} \mathrm{b}^{*}$ coordinates of the master and copy patches and returns their $\Delta E^{\text {mod }}$. The whole dataset of masters was first used to design and train the network, i.e., 1232 masters (see Section III-B).

1) Generating the training and test sets: The training set was created using the developed tool. In more detail, a total of 16 copies was generated for each master $m$ : four copies very close to the master $\left(0.5<\Delta E_{m, c} \leq 2\right)$, four copies close to the master $\left(2<\Delta E_{m, c} \leq 3\right)$, four copies a little far from the master $\left(3<\Delta E_{m, c} \leq 5\right)$, and the last four copies far away from the master $\left(5<\Delta E_{m, c} \leq 10\right)$. This led to 19712 master-copy pairs of $\mathrm{L}^{*} \mathrm{a}^{*} \mathrm{~b}^{*}$ coordinates, and made it possible to uniformly cover all the dataset, as can easily be seen in Fig. 11. Given a master $m_{i}$ and the $j$-th of its copies
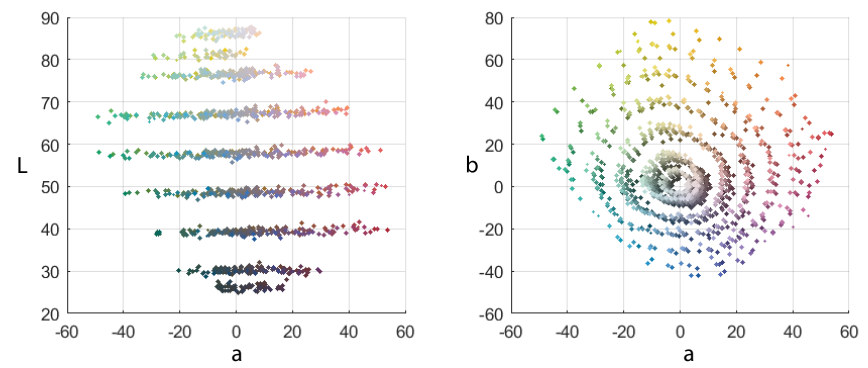

Fig. 11. Distribution of the data in the $\mathrm{L}^{*} \mathrm{a}^{*} \mathrm{~b}^{*}$ space.

$c_{i, j}$, the master-copy pair of $\mathrm{L}^{*} \mathrm{a} * \mathrm{~b} *$ coordinates is associated with its $\Delta E_{m_{i}, c_{i, j}}^{m o d}$, in order to obtain the 19712 samples to show to the network. The structure of the sample to use for 


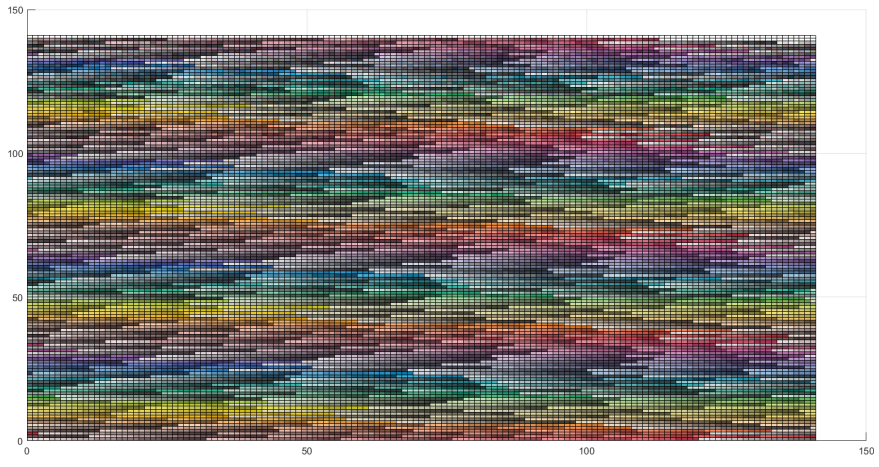

Fig. 12. Masters and copies used for the training. Each master is followed by its copies in the same row, from left to right.

the training is

$$
\text { sample }_{i}=\left(\left(L_{m_{i}}^{*}, a_{m_{i}}^{*}, b_{m_{i}}^{*}, L_{c_{i, j}}^{*}, a_{c_{i, j}}^{*}, b_{c_{i, j}}^{*}\right), \Delta E_{m_{i}, c_{i, j}}^{\text {mod }}\right),
$$

where $L_{m_{i}}^{*}, a_{m_{i}}^{*}, b_{m_{i}}^{*}$, and $L_{c_{i, j}}^{*}, a_{c_{i, j}}^{*}, b_{c_{i, j}}^{*}$ are the $\mathrm{L}^{*} \mathrm{a}^{*} \mathrm{~b}^{*}$ coordinates of master $m_{i}$ and copy $c_{i, j}$, respectively. The whole set of masters and their copies is shown in Fig. 12.

The test set was generated with the help of the developed graphic tool, with the same structure as the training set. In particular, a total of 900 master-copy pairs were uniformly selected from the $\mathrm{L}^{*} \mathrm{a} \mathrm{b}^{*}$ space, and then each pair was associated with its $\Delta E^{\text {mod }}$.

2) Neural network architecture: The neural network has six neurons in the input layer and one neuron in the output layer. The input is made up of two triples of neurons, which receive the $\mathrm{L}^{*} \mathrm{a}^{*} \mathrm{~b} *$ coordinates of master $m$ and copy $c$, respectively. The output is $\Delta E_{m, c}^{\text {mod }}$.

Multiple networks were trained in order to find the best architecture. More in detail, a single hidden layer was used, whose number of neurons was experimentally found varying it from 20 to 200 with step one. For each number of neurons, the network was trained ten times and the mean squared error (MSE) on the test set was calculated. The number of hidden neurons that produced the lowest MSE was chosen as the best one. The network was trained according to the procedure described above by separately using the Levenberg-Marquardt backpropagation and Bayesian regularization backpropagation training functions.

3) Results: The best result for the Levenberg-Marquardt training function was achieved with 50 neurons. On the other hand, with the Bayesian training function, the best result was achieved with 100 neurons.

The probability distribution of the error is shown in Fig. 13. As the figure shows, both networks achieve a good result, with errors that are very likely to range from -0.05 to 0.05 . This means that the networks are highly accurate. The network trained using the Bayesian training function was included into the neural system.

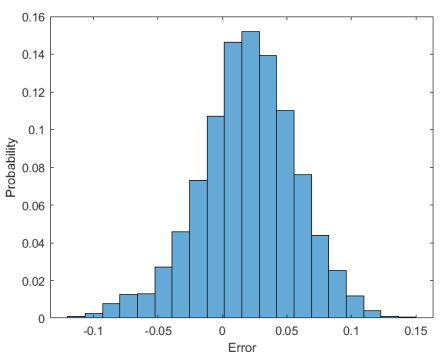

(a)

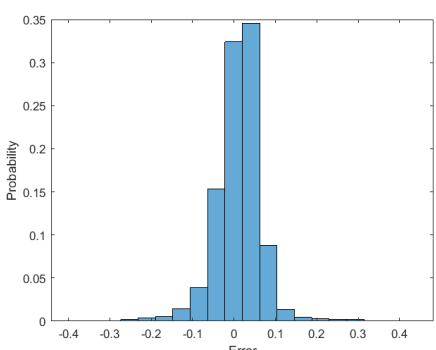

(b)
Fig. 13. Probability distribution of the error when using 50 neurons in the hidden layer and Levenberg-Marquardt training function (a), and when using 100 neurons in the hidden layer with Bayesian training function (b).

\section{E. Second comparator: improving $\Delta E_{\text {mod }}$}

This section describes a second neural network that was purposely designed and trained to include the human aspects related to the perception of colors described in Section III-C. This was done to improve the accuracy of the comparison when considering similar colors with slightly different hues.

1) Generating the training and test sets: The training set is made up of 2000 pairs of similar colors, i.e., with $\Delta E$ between 2 and 3 , and 800 pairs with $\Delta E$ between 3 and 5 . The distribution of the data is shown in Fig. 14 (i.e., the bold dots). As can be seen, colors are uniformly distributed over the color space. After generating these pairs of colors, they
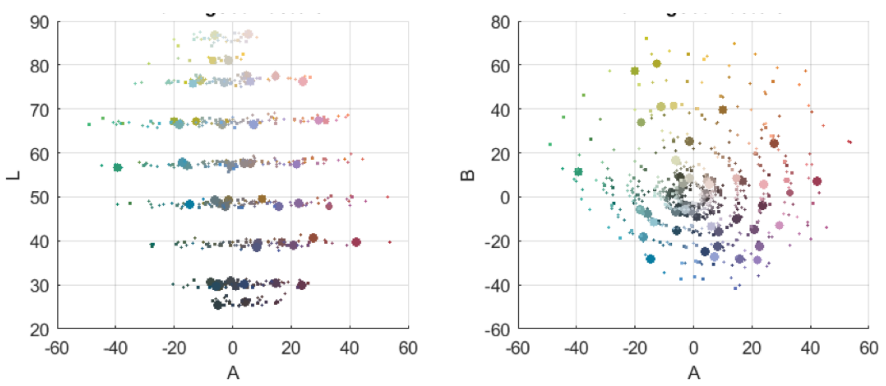

Fig. 14. Distribution of the data in the $\mathrm{L}^{*} \mathrm{a} \mathrm{b}^{*}$ space

were analyzed by ten observers who quantified the similarity level using the graphic interface of the system. The value of coefficient $k$ was then associated to the similarity levels as explained in Section III-C.

A total of 2800 samples with the following structure were thus obtained to train the network:

$$
\text { sample }_{i}=\left(\left(L_{m_{i}}^{*}, a_{m_{i}}^{*}, b_{m_{i}}^{*}, L_{c_{i, j}}^{*}, a_{c_{i, j}}^{*}, b_{c_{i, j}}^{*}\right), \sigma_{m_{i}, c_{i, j}}\right) .
$$

The test set was generated by using the developed graphic tool, with the same structure as the training set, i.e., made up of two groups of pairs, the first with $\Delta E$ between 2 and 3 , and the second with $\Delta E$ between 3 and 5 . A total of 200 mastercopy pairs were generated, by uniformly sampling them in the $\mathrm{L}^{*} \mathrm{a}{ }^{*} \mathrm{~b}^{*}$ space. Each pair was finally associated with the corresponding value of $\sigma$. 
2) Neural network architecture: The MLP neural network has six input neurons and one output neuron. The first three neurons of the input take the $\mathrm{L}^{*} \mathrm{a}^{*} \mathrm{~b}^{*}$ coordinates of master $m$, whereas the next three neurons receive the $\mathrm{L}^{*} \mathrm{a} * \mathrm{~b} *$ coordinates of copy $c$. The output is $\sigma_{m, c}$.

A diverse set of networks were trained, and this made it possible to find out the best architecture. As done for the network described in Section IV-D2, a single hidden layer was used. In order to find the best number of neurons to put in the hidden layer, different networks were tested by increasing the number of neurons in the hidden layer, starting from 20 up to 200, with step one. For each value, the corresponding network was trained ten times and the MSE on the test set was finally calculated. The network was trained using both the Levenberg-Marquardt backpropagation and Bayesian regularization backpropagation training functions.

3) Results: In this case, a definitely better performance was achieved by training the networks with the Bayesian regularization backpropagation. The network with 100 neurons in the hidden layer obtained the highest accuracy.

The probability distribution of the error is shown in Fig. 15. As can be seen, the error is just a little bit higher than zero. Values that are higher than 0.02 are very unlikely to obtain with this network, which can thus be reasonably considered as highly accurate in reproducing what the human eye perceives when observing two similar colors that come from an industrial printing process.

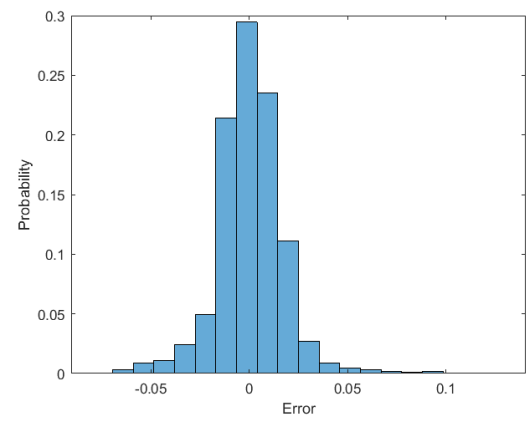

Fig. 15. Probability distribution of the error obtained by the best neural network to reproduce $\sigma$.

\section{COnClusions}

This paper has presented a neural network-based system that automatically compares two printed colors and returns a perceptually uniform measure of their difference as perceived by the human eye, without being affected by the typical phenomena that alter the perception of colors.

In order to achieve this result, the $\mathrm{L}^{*} \mathrm{a} * \mathrm{~b}^{*}$ distance has first been regularized to incorporate how the human eye actually perceives the different levels of brightness. By means of visual comparisons, the measure has then been refined to better map the human perception of the difference of colors.

The results have shown that the neural system-i.e., the two neural comparators-accurately compares two colors, and returns a measure whose value is actually higher the greater the difference the human eye perceives.

The system can thus be extremely useful for those workers who perform visual checks in industrial sectors where printing accurate colors is key, thereby providing them with an objective measure of the difference of two printed colors. The developed tool can also help establish the most accurate level of color tolerance of the production process. This lets the color vary a little bit, making the process cost-effective with no influence on the final quality that the customer perceives.

\section{ACKNOWKEDGMENT}

The authors would like to thank Roberto Ciardi, Alessandro Degiovanni and Giovanni Falzone for developing the system.

\section{REFERENCES}

[1] https://www.smitherspira.com. Worldwide authority on the packaging, paper and print industry supply chains.

[2] H. Foley and M. Matlin, Sensation and Perception. Psychology Press, 2015.

[3] A. Gilchrist and J. Nobbs, "Colorimetry, theory," in Encyclopedia of Spectroscopy and Spectrometry (Third Edition), third edition ed., J. C. Lindon, G. E. Tranter, and D. W. Koppenaal, Eds. Oxford: Academic Press, 2017, pp. $328-333$.

[4] A. D. Broadbent, "Colorimetry, methods," in Encyclopedia of Spectroscopy and Spectrometry (Third Edition), third edition ed., J. C. Lindon, G. E. Tranter, and D. W. Koppenaal, Eds. Oxford: Academic Press, 2017, pp. $321-327$.

[5] S. Billmeyer, Principles of Color Technology. New York: Wiley \& Sons, 2000

[6] H. Yu, T. Cao, B. Li, R. Dong, and H. Zhou, "A method for color calibration based on simulated annealing optimization," in $20163 \mathrm{rd} \mathrm{In-}$ ternational Conference on Information Science and Control Engineering (ICISCE), July 2016, pp. 54-58.

[7] Y. Wang, X. Wang, and J. Li, "Color appearance phenomena under high ambient illumination," Optik - International Journal for Light and Electron Optics, vol. 145, pp. 22 - 29, 2017. [Online]. Available: http://www.sciencedirect.com/science/article/pii/S0030402617307878

[8] M. Ataeefard, "Investigating the effect of paper properties on color reproduction of digital printing," Progress in Organic Coatings, vol. 77, no. 9, pp. 1376 - 1381, 2014.

[9] H.-J. Kwon, S.-H. Lee, G.-Y. Lee, and K.-I. Sohng, "Luminance adaptation transform based on brightness functions for ldr image reproduction," Digital Signal Processing, vol. 30, pp. 74 - 85, 2014.

[10] A. P. Perroni, C. D. Bergoli, M. B. F. dos Santos, R. R. Moraes, and N. Boscato, "Spectrophotometric analysis of clinical factors related to the color of ceramic restorations: A pilot study," The Journal of Prosthetic Dentistry, vol. 118, no. 5, pp. 611 - 616, 2017.

[11] J. M. Medina and J. A. Daz, "Classification of batch processes in automotive metallic coatings using principal component analysis similarity factors from reflectance spectra," Progress in Organic Coatings, vol. 88, pp. $75-83,2015$. 